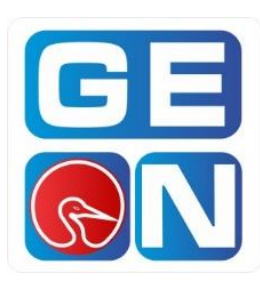

Revista GEON (Gestión, Organizaciones y Negocios.) ISSN: 2346-3910 en línea revistageon@unillanos.edu.co Universidad de los Llanos Colombia

Riveros Polania, Gustavoi; Pedraza, Camiloii; Barrero, Yessica ${ }^{\mathrm{iii}}$; Aguilar, Ana María ${ }^{\text {iv }}$.

Categorización de los establecimientos según los valores agregados percibidos por el consumidor en niveles socioeconómicos 3 y 4 en Bogotá, Colombia

Revista GEON, Vol. 6, No. 2, 2019 Pág. 63-81

Disponible en: https://doi.org/10.22579/23463910.156

\footnotetext{
${ }^{\mathrm{i}}$ https://orcid.org/0000-0002-4781-9836

ii https://orcid.org/0000-0002-3371-0602

iii https://orcid.org/0000-0002-6508-1150

iv https://orcid.org/0000-0002-9747-1655
}

Esta publicación se encuentra bajo licencia: Creative Commons ReconocimientoNoComercialSinObraDerivada 4.0 Internacional

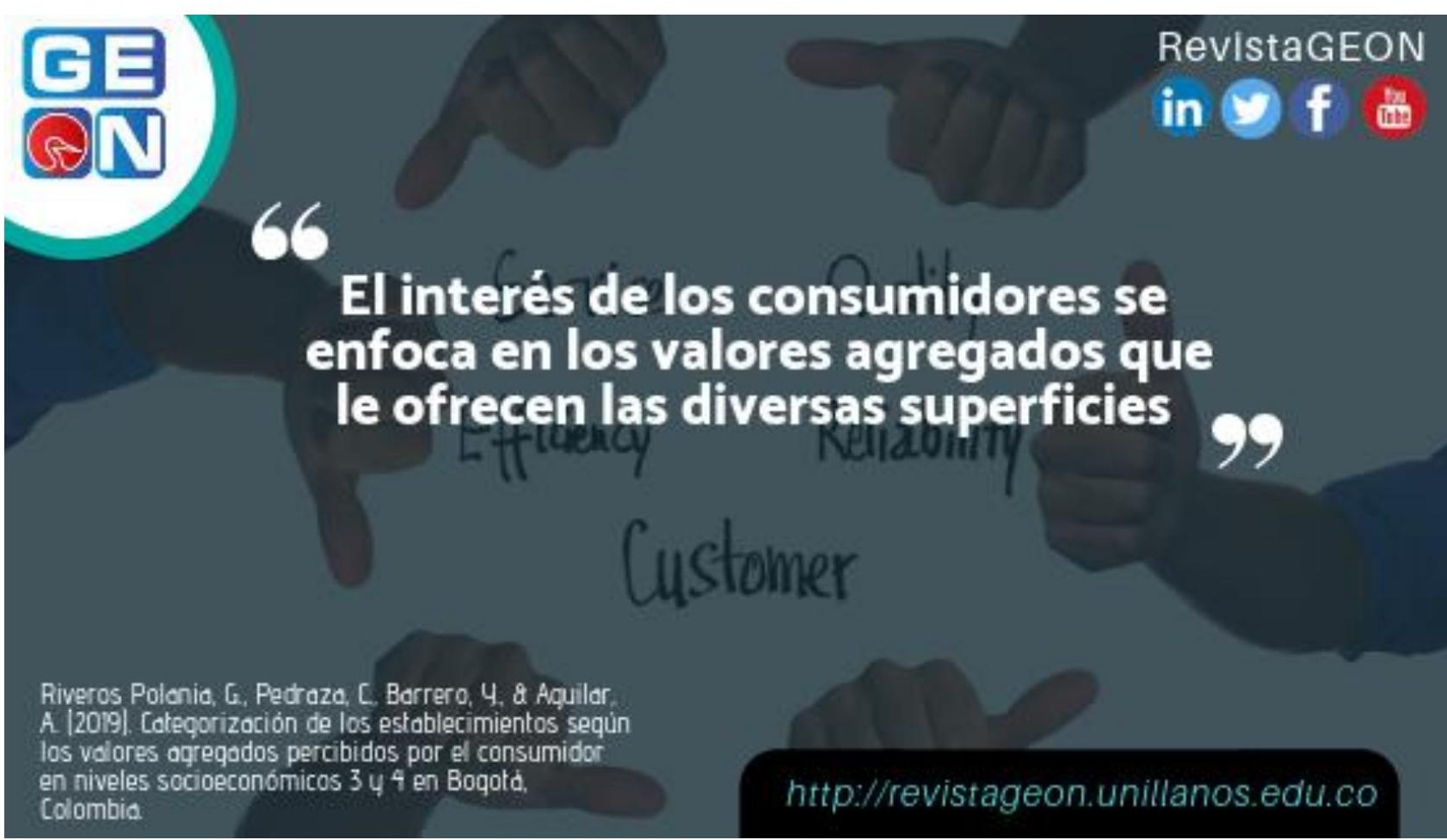




\title{
Categorización de establecimientos por los valores agregados percibidos de consumidores de niveles socioeconómicos 3 y 4 en Bogotá, Colombia
}

\section{Categorization of retail by perceived added values from consumers of socioeconomic levels 3 and 4 in Bogotá, Colombia}

\section{Gustavo Riveros $^{1}$-Camilo Pedraza ${ }^{2}$-Yessica Barrero-Ana María Aguilar ${ }^{4}$}

Cómo citar este Riveros Polania, G., Pedraza, C., Barrero, Y., \& Aguilar, A. (2019). Categorización de los establecimientos artículo / To según los valores agregados percibidos por el consumidor en niveles socioeconómicos 3 y 4 en Bogotá, reference this Colombia. Revista GEON (Gestión, Organizaciones Y Negocios), 6(2), 63-81. Recuperado a partir de article:

http://revistageon.unillanos.edu.co/index.php/geon/article/view/156

\begin{abstract}
Resumen
Artículo de investigación:

Fecha de recepción: $2019 / 05 / 20$

Fecha de aceptación: $2019 / 06 / 30$

Esta publicación se encuentra bajo licencia: Creative Commons

Reconocimiento-

NoComercial-

SinObraDerivada 4.0

Internacional.

El presente artículo es resultado de un proyecto de investigación, que tuvo como objetivo principal identificar la categorización de los canales de distribución de retail, de acuerdo a los valores agregados percibidos por consumidores de niveles socioeconómicos 3 y 4 de la localidad de Suba, en la ciudad de Bogotá. Se realizó una investigación cualitativa de tipo exploratoria, con la aplicación de muestreo no probabilístico por bola de nieve e instrumentos de recolección de datos mediante entrevistas semiestructuradas y grupos focales, que permitieron la identificación de los valores percibidos por los consumidores frente a los productos y servicios ofrecidos por los establecimientos.
\end{abstract}

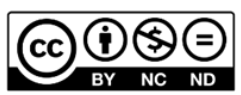

\footnotetext{
${ }^{1}$ Docente Investigador, Facultad de Mercadeo, Director de Semillero de Investigación en Retail: Gustavo Riveros Polanía, Economista y Administrador de Empresas, Master en Gerencia Estrategia de Mercadeo / Universidad Santo Tomás Bogotá / gustavoriveros@usantotomas.edu.co, https://orcid.org/0000-0002-4781-9836

2 Estudiante de Mercadeo, auxiliar de investigación: Camilo Pedraza, Universidad Santo Tomás / camilo.pedraza@usantotomas.edu.co, https://orcid.org/0000-0002-3371-0602

${ }^{3}$ Estudiante de Mercadeo, auxiliar de investigación: Yessica Barrero, Universidad Santo Tomás / yessicabarrero@usantotomas.edu.co, https://orcid.org/0000-0002-6508-1150

${ }^{4}$ Estudiante de Mercadeo, auxiliar de investigación: Ana María Aguilar, Universidad Santo Tomás / anaaguilar@usantotomas.edu.co, https://orcid.org/0000-0002-9747-1655
} 
Los resultados indicaron que valores como calidad, ubicación, promoción, oferta y servicio, representan mayor importancia en el conocimiento de la clase de establecimiento retail visitado, de tal manera, que los consumidores no identifican una clasificación de los intermediarios y por el contrario, tienen en cuenta los valores mencionados.

Palabras Clave: Canales de distribución, valores percibidos, categorización de establecimientos, consumo..

\section{Código JEL: 0540}

\section{Abstract}

This article is the result of a research, whose main objective was to identify the category of retail distribution channels, according to the added values perceived by consumers of socioeconomic levels 3 and 4 of Suba District in Bogota. It was carried an exploratory qualitative investigation out, with the application of non-probabilistic snowball sampling and data collection instruments through semi-structured interviews and focus groups, which allowed the identification of perceived values by consumers in front of products and services offered by the establishments.

The results indicated that values such as quality, location, promotion, offer and service, represent greater importance than the knowledge of the kind of retail establishment visited, so that consumers do not identify a classification of intermediaries and, on the contrary, they consider the mentioned values.

Keywords: Distribution channels, perceived values, categorization of establishments, consumption.

Code JEL: O540

\section{Introducción}

El continuo interés en el mercadeo por conocer la percepción de los consumidores acerca del precio, atributos y beneficios, influye de manera determinante en su intención de compra y consumo ( Lazar Knuk \& Schiffman, 2005), siendo estos algunos aspectos que inquietan a los empresarios; por ello, es importante abordar la temática de canales de distribución y la percepción que tiene el consumidor sobre los mismos, para fortalecer las estrategias sobre los atributos más relevantes del mismo establecimiento de acuerdo a su categorización. 
Esto permite que los canales de distribución brinden a los fabricantes, intermediarios y consumidores la suficiente información sobre los beneficios que se tendrán con relación al tiempo y al lugar, logrando que los productos y servicios estén disponibles para el consumidor en el momento preciso, las condiciones y precio adecuados y en el lugar conveniente, para la satisfacción de sus necesidades. (Kotler \& Armstrong, 2003).

Desde allí, la importancia de evaluar los valores agregados que percibe el consumidor frente a las diferentes categorías de establecimientos de distribución retail existentes al momento de la decisión de compra. La población objeto de estudio, estuvo centrada en consumidores de niveles socioeconómicos 3 de 4 ubicados en la Localidad de Suba de la ciudad Bogotá. Actualmente, Bogotá cuenta con 7.181.469 de acuerdo al Censo DANE de 2018, distribuidos en 8 localidades: Usaquén, Chapinero, Santafé, San Cristóbal, Usme, Suba y Ciudad Bolívar. La Localidad de Suba tenía una población de 923.064 según Censo DANE de 2005 y los informes señalan una población proyectada a 2020 de 1.381.597, representada por una tasa de crecimiento poblacional de $2.70 \%$. (Secretaría Distrital de Planeación de Bogotá, 2019).

Suba, es una localidad que cuenta con 12 Unidades de Planeamiento Zonal (UPZ), ubicadas en un área de 6270,7 has., y "de acuerdo al POT (Plan de Ordenamiento Territorial), tienen como propósito definir y precisar el planeamiento del suelo urbano, respondiendo a la dinámica productiva de la ciudad y a su inserción en el contexto regional, involucrando a los actores sociales en la definición de los aspectos de ordenamiento y control normativo a escala zonal" (Secreataría Distrital de Planeación de Bogotá, 2019).

Objetivos. Consistieron en un objetivo generar orientado a identificar la categorización de canales de distribución de retail según los valores agregados percibidos por los consumidores de niveles socioeconómicos 3 y 4 de la localidad de Suba en la ciudad de Bogotá. Como objetivos específicos se planteó analizar la categorización de canales de distribución de retail por medio de la percepción de los consumidores; identificar las diferentes características de los establecimientos de retail teniendo en cuenta el comportamiento de compra de los consumidores, y por último, conocer los valores agregados que ofrecen los establecimientos a los consumidores.

Problemática y justificación. Hoy en día se observa un continuo interés en el mercadeo por conocer la percepción que tienen los consumidores acerca del precio, atributos y beneficios que influye de manera determinante en su intención de compra y consumo ( Lazar Knuk \& Schiffman, 2005) siendo estos algunos aspectos que inquietan los empresarios, por ello es importante abordar la temática de los canales de distribución y la percepción que el consumidor tiene de ellos para lograr fortalecer los atributos relevantes que lleven a la categorización del mismo. 
Según el Presidente de America Retail, Willem F. Schol, se está presentando una evolución a nivel cliente - consumidor, caracterizado por ser más exigente, empoderado, y que requiere muchas alternativas a la hora de comprar, así como le necesidad de oferta de soluciones personalizadas y la preocupación y cuidado del medio ambiente. Este consumidor heterogéneo representan cada uno un segmento importante en el mercado, lo que cambia son los factores que influyen en su preferencia de compra (America Retail, 2019).

Así mismo, los avances tecnológicos cambiar la forma de vender y acceder a los productos, la robótica, la realidad virtual y aumentada y, la inteligencia artificial son herramientas que están cambiando la forma y preferencia del proceso de compra y consumo. De esta forma los establecimientos de retail, están afrontando el reto de ofrecer soluciones de valor real, teniendo en cuenta la conveniencia de los clientes y de la de la empresa, de tal forma, que deben ofrecer variedad de alternativas para los clientes además de tiendas físicas, comercio electrónico, redes sociales, market places, entrega a domicilio, recojo en la tienda (America Retail, 2019).

La tendencia hacia la compra basada en la experiencia se vuelve de esa manera, en el centro de interés de los establecimientos de retail. Los clientes están en la búsqueda de que su compra sea mucho más, que una simple transacción. Esta experiencia no solo tiene que ver con el lugar y forma de compra, sino con una mayor rapidez a través de internet o de puntos físicos, facilidad y rapidez de cambio en caso de inconvenientes y amplios horarios de atención de las tiendas, por las limitaciones de tiempo para planear sus comprar, posibilidad y seguridad de los pagos en línea y desde teléfonos móviles e incentivos, descuentos y estrategias de recordación de compra; todo esto al final definen la diferenciación de los retailers para su éxito en el mercado (America Retail, 2019).

De todo lo anterior, se evidencia la dinámica, desarrollo y crecimiento de los establecimientos de retail en Colombia. Según reporte de America Retail (2019), en 2018 se registró movimientos por al menos 80 billones de pesos por parte de los supermercados en Colombia, de los cuales Almacenes Éxito se ubicó en primer lugar con 55 billones (63,3\%), seguido por Olímpica con 6,1 billones (7,4 \%), Alkosto con 5,7 billones $(6,9 \%)$, D1 con 3,79 billones $(4,5 \%)$, Cencosud con 3,77 billones (4,5\%), Ara con 2,2 billones (2,7 $\%)$. En séptimo lugar, se ubicó Justo y Bueno con 1,6 billones (1,9\%), seguido por Makro con 1,3 billones (1,5\%), Price Smart con 1,19 billones $(1,4 \%)$ y La 14 con 1,12 billones (1,3\%) (America Retail, 2019). Según la misma fuente, que los almacenes de descuento reportaron aumentos en sus ventas, D1 reportó un 22 $\%$, Ara un $53 \%$ y Justo y Bueno un $113 \%$ de crecimiento.

Contexto Teórico. Para el abordaje del tema de investigación, se abordaron temáticas relacionadas con percepción, consumidor, canales de distribución y establecimientos de retail. 
Percepción. Según Lazar Knuk \& Schiffman (2005) "la percepción es un proceso mediante el cual un individuo selecciona, organiza e interpreta los estímulos para formarse una imagen significativa y coherente del mundo" ( $\mathrm{p}$. 17), por esta razón la percepción es un proceso en donde la persona antes de clasificar subconscientemente la información sienta su entendimiento en las nociones previas que se poseen, de esta manera consecuentemente constituye un esquema que delimita y puede aceptar o rechazar un nuevo estímulo recibido (Rivera, Arellano, \& Molero, 2000).

Por ello se dice que la primera impresión concibe la relación entre canal y cliente, inicialmente el individuo genera perspectivas y análisis a priori sobre la superficie en donde adquiere productos y marcas, el segundo momento es la ejecución de sus compras en donde se involucra directamente con el lugar y amplía su visión sobre la distribución espacial de los productos, la iluminación y la prioridad que da el almacén a cada ítem específico; si bien esta clasificación de información no se hace a nivel consciente, se determina la intención de la adquisición final del cliente en donde se crea la "percepción experimental" que define la categorización a posteriori que hace el consumidor frente al canal de distribución.

Por esta razón se crea un concepto de organización en la mente de cada consumidor, lo cual nos lleva a entender que la percepción de una organización debe ser fortalecida con lo que desea transmitir cada compañía para lograr una visión estable de las cualidades y características de las mismas. (Capriotti, 1992)

Para Céspedes, Cortés, \& Madrigal (2011) existen dos alternativas para medir la percepción del consumidor, tales como la validación interna, que consiste en la capacidad del instrumento que se aplica para medir todas las dimensiones de la percepción del consumidor e indica el grado de homogeneidad encontrada y la validación externa, que se refiere al juicio de los resultados de acuerdo con la proporción en que cada una de las variables de percepción seleccionadas puedan explicar el comportamiento del consumidor de forma general.

Es importante tener en cuenta, que una medición para validación interna, se presenta cuando la respuesta corresponde a más de dos opciones, lo que permite identificar con más facilidad la clasificación adecuada de las variables que definen los procesos de cada canal o retailer en un momento determinado para facilitar el proceso de compra de los consumidores.

Consumidor. El consumidor se define como una "persona física o jurídica que, actuando en un ámbito ajeno a una actividad empresarial o profesional, adquiere, utiliza o disfruta bienes, productos, servicios, actividades $\mathrm{o}$ funciones para uso personal, familiar y colectivo, siempre que lo haga como destinatario final, y siendo indiferente que quienes producen, facilitan, suministran o expiden dichos bienes o servicios tengan naturaleza pública o privada, individual o colectiva." (Real Aacademia Española, 2019) 
Dentro del papel del comportamiento del consumidor se evidencia una clasificación en dos, el personal que es aquella persona que frecuenta una compra individual de bienes o servicios y se destaca por ser el famoso consumidor final, y el organizacional que está basado en empresas $u$ organizaciones con propósito de lucro, las cuales compran bienes, servicios y/o equipos para mantener el desarrollo de la organización (Lazar Knuk \& Schiffman, 2005).

Por dichas razones es necesario conocer al consumidor para saber sus necesidades y sus actitudes a la hora de ejercer un rol de compra en el cual será más fácil entender la influencia de sus acciones en el punto de venta, cabe resaltar que en este proceso se ejercen cinco figuras representativas que se pueden evidenciar cómo una misma persona, las cuales se describen en: Iniciador, influenciador, decisor, comprador, usuario (Molero \& Rivero, 2009).

Según un estudio de Fanalco (2012), los empresarios deben asumir el cambio acelerado del comportamiento del consumidor en Colombia está cambiando con relación a hace 30 años, los cuales destinaban sus presupuestos para adquirir en su mayoría productos del sector de alimentos, mientras que hoy por hoy, se enfocan a educación, comunicaciones y transporte. Estas tendencias sobre el comportamiento de compra generan oportunidades para el mercado aprovechando mayores rentabilidades (Fenalco, 2012).

Canales de distribución. Los canales de distribución $\mathrm{Se}$ definen como redes interorganizacionales $\mathrm{O}$ superorganizaciones, que permiten los flujos de productos desde productores hasta los usuarios industriales $\mathrm{o}$ consumidores. Los canales de distribución consisten en una red de socios entre productores y consumidores, incluidos los intermediarios, como mayoristas $y$ minoristas o establecimientos o retailers, que integran y facilitan la ruta para el traslado de bienes de productores a usuarios industriales o consumidores. El propósito de los canales de distribución se establece es la facilitación y superación de brechas entre los lugares de producción y los lugares de consumo intermedio y consumo final que conducen a proporcionar servicios $\mathrm{o}$ productos adecuados al mercado (Andjelkovic, Barac, \& Radosavlje, 2017).

Para la American Marketing Association (A.M.A) los canales de distribución son "una red organizada (sistema) de agencias e instituciones que, en combinación, realizan todas las funciones requeridas para enlazar a productores con los clientes finales para completar las tareas de marketing". Estos canales de distribución, se dice que son "el conjunto de organizaciones y funciones interdependientes involucradas en el proceso de poner un bien o servicio a la disposición de sus usuarios o consumidores" (Frederick Ross, 2012),

Las estrategias de los canales de distribución representan el diseño y gestión de las relaciones entre socios como uno de los factores de competitividad, de acuerdo a las características de franqueza, niveles, densidad, variedad y novedad. La franqueza implica el contacto directo entre 
productores y consumidores sin involucrar a intermediarios. Los canales de distribución pueden ser cortos o directos y largos o indirectos, dependiendo de los factores como las características del mercado, los consumidores, los productos o las materias primas, las relaciones entre los socios en el canal, etc. Los niveles del canal dependen del número de diferentes socios de compra y venta, que existen entre productores y consumidores. (Andjelkovic, Barac, \& Radosavlje, 2017).

La importancia de los canales de distribución, radica en que proporcionan disponibilidad de productos en el mercado, cooperación y colaboración en la red, concentración de empresas de acuerdo con las actividades centrales, un nivel de servicio apropiado, un mínimo de costos logísticos y totales, el intercambio de información precisa y confiable en flujos directos e inversos, y la eficiencia transaccional como resultado de un número reducido de conexiones $\mathrm{y}$ actividades. Todo lo anterior, se conjuga para superar las diferencias de espacio, tiempo, calidad y cantidad, entre el lugar de producción y el lugar de consumo. Además, a través de los canales de distribución hay diferentes flujos: productos, información y flujo financiero (Andrejić, 2015, 22 citado por Andjelkovic, Barac, \& Radosavlje, 2017).

De acuerdo a estudios sobre percepción del consumidor sobre los canales de distribución, las visiones de los establecimientos de retail son caracterizadas por la brevedad, la claridad, la orientación futura, la estabilidad, el desafío, la abstracción y la capacidad de inspirar y contener referencias a las ventas, el consumidor, los empleados y el liderazgo del mismo establecimiento que representan el liderazgo mejorado de la gerencia percibida por los involucrados. Estas visiones además predicen indirectamente una mejor satisfacción del personal, mientras que el liderazgo percibido del personal mejora directamente la satisfacción del personal. No hay un efecto directo de la satisfacción del personal sobre la satisfacción del consumidor como se encuentra normalmente en otros lugares (Kantabutra, 2011).

De esta forma, el impacto generado por el compromiso, la confianza y las relaciones dentro de los canales de distribución, tienen un impacto significativo en la relación sólida y a largo plazo entre los socios del canal. Los estudios futuros sobre la gestión de canales se seguirán enfatizando, más en la gestión de las relaciones entre personas, empresas $y$ organizaciones que realizan funciones de distribución, orientadas a crear valor para los clientes en cuanto a la calidad del producto y su disponibilidad en el momento y lugar correctos (Frazier, 1999).

La evolución de los canales de distribución afecta a todo el mundo, partiendo con los distribuidores que iniciaron con grandes edificios en las ciudades, luego pasaron a tiendas especializadas en centros comerciales y más tarde a formatos de grandes superficies en las que se ofrecen amplias secciones de artículos rebajados, llegando a la transformación más profunda que ha experimentado el sector de la distribución, 
en donde se perciben cambios significativos en el modo, lugar y momento en el que compran los consumidores (IBM, 2012).

Cada intermediario se puede categorizar según las características particulares del canal o retailer. Entre los intermediarios que posee mayor cantidad de clasificaciones son los detallistas, minoristas o establecimientos retail, en donde se identifican las tiendas tradicionales, los centros comerciales, los establecimientos de autoservicio, los supermercados, los grandes almacenes, Las tiendas de descuento y los outlets (Sainz de Vicuña Ancín, 2000).

\section{Materiales y métodos}

Se elige una investigación de enfoque cualitativo en donde se busca conocer a profundidad el tema que se está abordando (Sampieri, Collado, Lucio, \& Perez, 1998), a partir de un estudio de tipo exploratorio-descriptivo, teniendo como base la aplicación de técnicas cualitativas como la entrevista semiestructurada dirigida a consumidores.

La unidad de información o población a tener en cuenta son los consumidores de estratos socioeconómicos 3 y 4 de la localidad de Suba, teniendo una estratificación por vivienda, en donde se clasifican como estratos medios que albergan a los usuarios con recursos promedios. Esta clasificación es la aproximación a la diferencia socioeconómica jerarquizada en Bogotá (DANE, 2013).
Es importante señalar, que por el tipo de investigación, el muestreo fue no probabilístico, lo que conllevó a la toma de una muestra de personas por método de conveniencia. Los participantes se seleccionaron al azar, con el fin de obtener información veraz y de mayor cubrimiento. Los participantes entrevistados, correspondieron a personas adultas, mayores de 25 años, que frecuentan los establecimientos comerciales ubicados en sitios estratégicos de la localidad de Suba.

Se indagaron tres dimensiones o focos de interés: a) percepción de los consumidores; b) categorización de los canales de distribución y c) Características y valores agregados de compra y consumo; los cuales proporcionaron la información suficiente para determinar lo que realmente piensan los consumidores.

El análisis de los datos se llevó a cabo a través del programa Atlas Ti en donde se realizó un análisis de los resultados cualitativos de los datos recogidos. Los materiales utilizados fueron cartillas desarrolladas con el investigador principal donde se planteó la metodología de las entrevistas, además de equipos de cómputo especializados para el manejo del programa Atlas Ti.

\section{Resultados y discusión}

La red semántica-experiencial en el programa Atlas TI, permite mostrar, como la mayoría de las personas que se entrevistaron, sienten un gusto al comprar gran diversidad de productos que de una $u$ otra manera les satisfacen las necesidades 
sentidas. Tal como dijo el Participante CI 1:44 "me gusta, me hace sentir bien si encuentro lo que quiero". Otro grupo de personas sienten una incomodidad, debido a la situación económica actual, es decir, varios productos están a un precio poco asequible, CI 1:136 "es estrés por que el dinero cada vez alcanza menos. Es estresante".

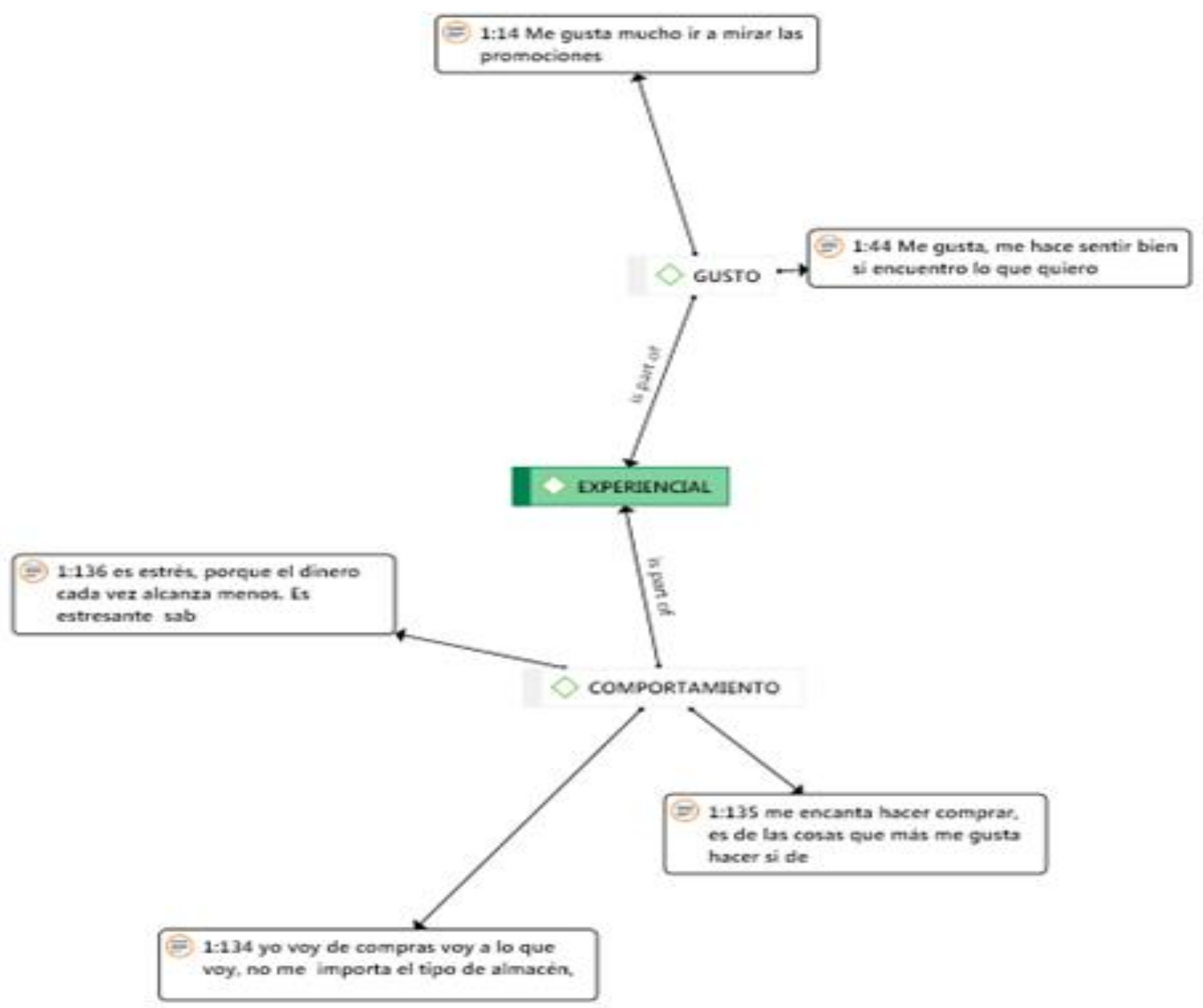

Figura 1: Red semántica experiencial.

Autor: Elaboración propia

Por el contrario, la gran mayoría de estas personas sienten afinidad y gusto a la hora de dirigirse hacia las tiendas de barrio, de descuento o tiendas de conveniencia, buscando la facilidad de conseguir gran variedad de productos sin preferencia alguna. CI 1.135 "Me encanta hacer compras, es de las cosas que más me gusta hacer.”. El público objetivo al que va dirigido este estudio, descubre los 
comportamientos de dichos participantes específicamente en la localidad de Suba en el estrato socioeconómicos 3 y 4 , de la ciudad de Bogotá, donde están ligados a los comportamientos de los familiares afines a la hora de dirigirse hacia un establecimiento, utilizando el "voz a voz".

Continuando con el análisis realizado en Atlas TI; un grupo de participantes que se entrevistaron sienten gusto y afinidad al hacer sus respectivas compras, lo hacen por satisfacción y así mismo, encontrar gran diversidad de productos a precios accesibles, sin importar el almacén que sea. CI 1:134 "Yo voy de compras, voy a lo que voy, no me importa el tipo de almacén". Uno de los factores importantes que se investigaron, es que la gran mayoría de estas personas no conocen el término de tiendas de descuento, pero sí se dirigen a ellas a realizar sus compras. En el análisis experiencial, vimos que los participantes no tienen afinidad hacia las marcas de establecimientos reconocidos como lo son: Éxito, Jumbo, Carulla, y demás.

Una vez desarrollado el análisis Experiencial, que la mayoría de personas sienten interés acerca de que son las tiendas de barrio, tiendas de descuento y tiendas de conveniencia, que como se dijo anteriormente, los entrevistados sienten gusto y tienen un comportamiento positivo a la hora de dirigirse hacia estos establecimientos, además, sienten placer por sus necesidades satisfechas, como lo demuestra los factores de importancia dentro de este análisis, como son: el gusto, afinidad, placer y comportamiento.

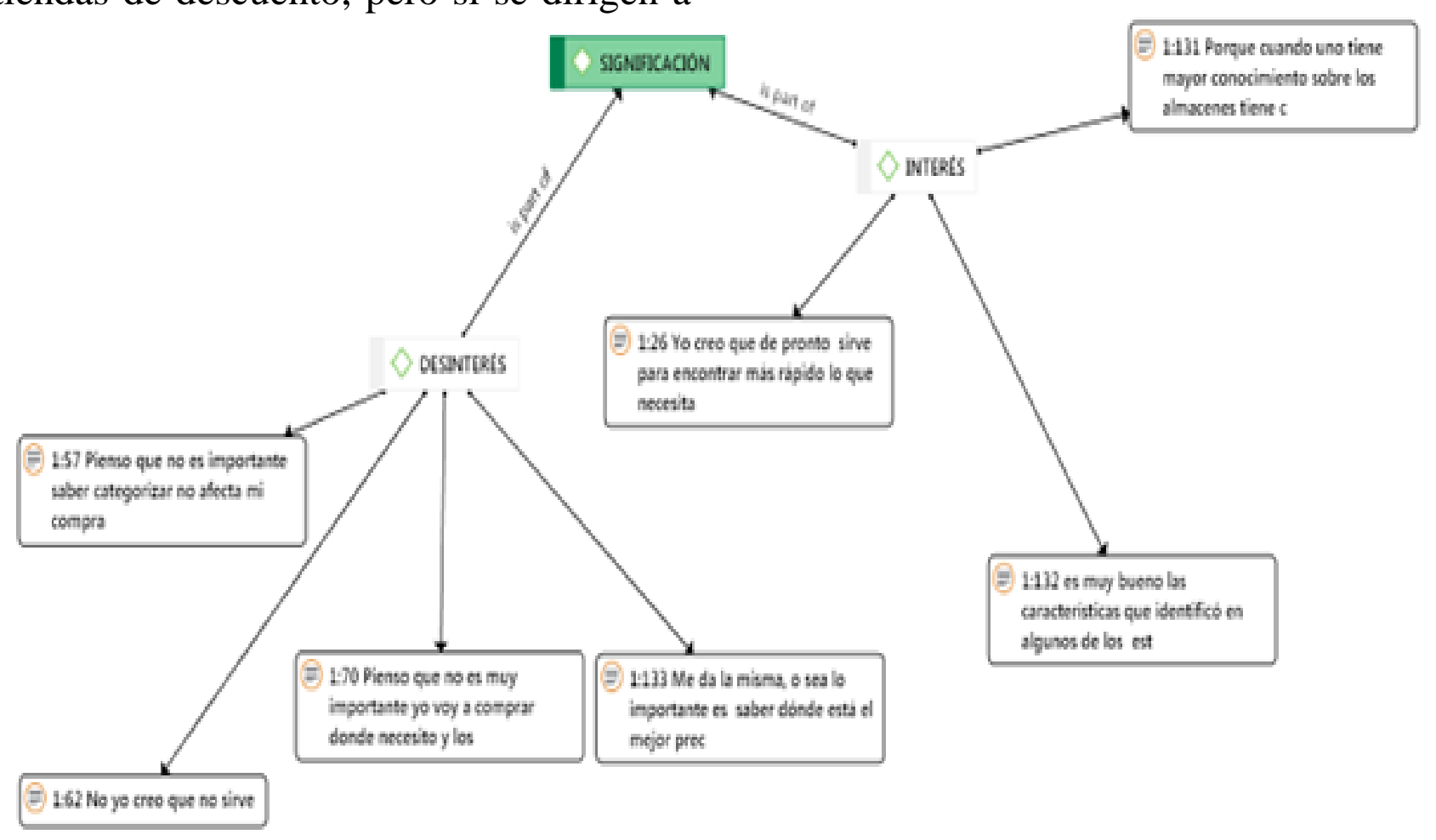

Figura 2: Red semántica significación. Autor: Elaboración propia 
Teniendo en cuenta la codificación de los datos textuales a través de atlas TI, se puede identificar que para los consumidores el hecho de clasificar los establecimientos no tiene gran significado porque para ellos es algo que no va a intervenir en su decisión de compra. En la red semántica de significación, se centraron dos variables que hacen referencia al interés y desinterés que puede llegar a tener el consumidor de estratos $3 \mathrm{y}$ 4 de la localidad de Suba sobre el tema.

Según los entrevistados, hay otras características que influyen en la decisión de compra: "Pienso que no es importante saber categorizar no afecta mi compra" CI:1:57; para este participante no hay importancia o relevancia de los términos o la categorización dada a los establecimientos, para ellos es algo que va inherente al momento de realizar la compra o el consumo. Este participante asocia con mayor importancia otras características que definen mejor lo que a él realmente le interesa saber cuándo va de compras, es decir, los valores agregados que ofrece el establecimiento.

Otro ejemplo, es la respuesta dada por el participante "me da la misma, o sea, lo importante es saber dónde está el mejor precio" CI:1:70, el cual hace referencia a un valor agregado en específico (el precio) dejando de lado, la importancia o relevancia que debería tener la categorización.

Por otro lado los participantes muestran un nivel de interés muy bajo en el conocimiento de las características que personaliza la categorización, dejando de lado su percepción, dicho de otra manera, hay algunas y muy escasas características que exigen un conocimiento de marca del establecimiento, a saber: uno de los participantes asegura "Yo creo que de pronto sirve para encontrar más rápido lo que se necesita" CI: 1:26, particularidades como estas son las que los participantes asocian con el aprendizaje o la conciencia de adquirir este tipo de conocimiento.

Igualmente, no todas las respuestas dadas por los participantes de estratos $3 \mathrm{y}$ 4 de la localidad de suba hicieron una negativa referencia a la importancia de la categorización, algunas personas también opinaron que podría ser bueno conocerla, pero por los beneficios que podría tener al identificar por ejemplo la marca, su lugar de ubicación, servicio al cliente y demás. Los valores agregados tomados como foco de análisis fueron seis: Calidad, Ubicación, Promociones, Oferta, Precio y Servicio. En la Calidad, los consumidores hacen referencia a que dentro de los establecimientos les gusta encontrar una relación adecuada entre la oferta de valor y el precio, el surtido y exhibición y por sobre todo la presentación, su calidad, como lo aseguró el consumidor de la entrevista 1:19 "Calidad a precios bajos, la calidad es buenísima.

La Ubicación, posee relevancia en la medida de la cercanía a los lugares residenciales en donde haya alta densidad poblacional, es decir que es muy importante tener inmediatez en cuanto a los productos de primera necesidad, así lo afirmó el participante de la entrevista 1:68 "yo creo que siempre están en lugares donde hay muchas viviendas cercanas"

Las Promociones son de gran interés. El participante 1:14 dice "Me gusta mucho ir a mirar las promociones" de esta forma cuando un almacén desee llamar la atención puede hacer promociones, 
además, que permite fidelizar a los consumidores frente a los productos, sus marcas y a la vez sobre los servicios de los establecimientos

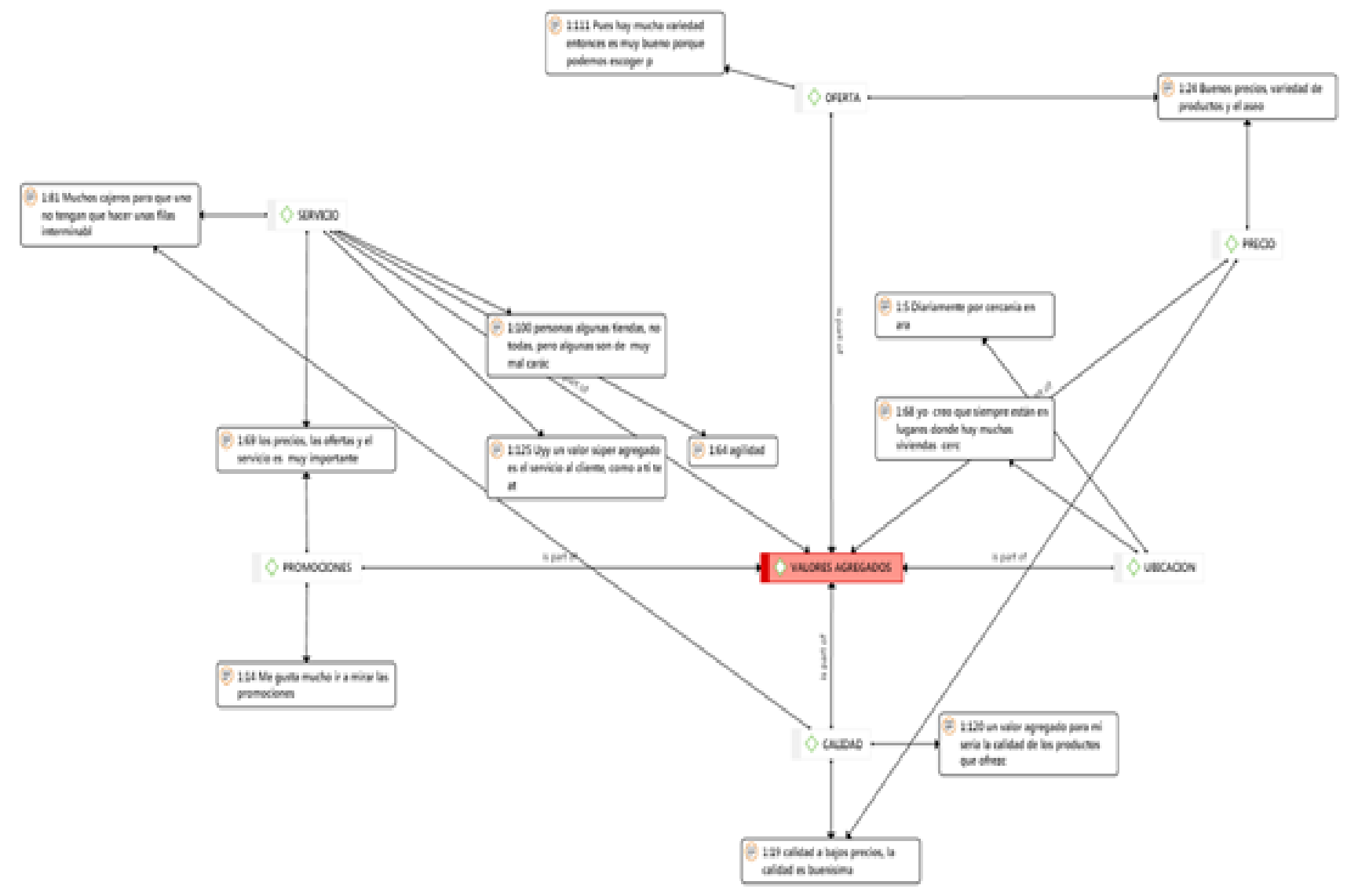

Figura 3: Red semántica valores agregados.

Autor: Elaboración propia

En cuanto a Oferta, se hace referencia al "Valor Ofertado" es decir a la variedad dentro del canal y también la pluralidad de los mismos canales de distribución. El participante de la entrevista 1:111 dice "Pues hay mucha variedad entonces es muy bueno porque podemos escoger dependiendo la necesidad". Pudimos evidenciar que a las personas les gusta que exista una amplia exhibición con distintas marcas, tamaños y opciones en general, así tienen la posibilidad de escoger aquello que se ajuste mejor a sus requerimientos y consiguientemente se abre la brecha para que los consumidores prueben productos nuevos y vayan descubriendo sus preferencias de diferentes clases de establecimientos.

El Precio es tomado por los consumidores en dos sentidos, en el primero, si es muy bajo, la calidad es dudosa y en segundo lugar si es muy alto, la marca es abusiva. De esta forma se determinó que, según las redes semánticas, el precio debe ser justo con el posicionamiento que posea el producto $\mathrm{y}$ el establecimiento. 
El Servicio es tomado no me parece”. Según las personas, en los fundamentalmente como que a las establecimientos los tenderos en ocasiones personas les gusta que no falte personal no tienen buen carácter y atienden de mala que ofrezca atención como lo afirma el participante de los outlets 1:125 "Uy un valor súper agregado es el servicio al cliente, como a ti te atienden", además que esté de buen carácter como dijo otro participante en la cita 1:100 "personas algunas tiendas, no todas, pero algunas son de muy mal carácter y hacen caras que forma, se concluye, que las personas a cargo de un establecimiento y contacto con los clientes deben ser capacitadas. El servicio debe ser útil, de manera rápida y que no genere desperdicio de tiempo, aprovechando la fidelización de los clientes.

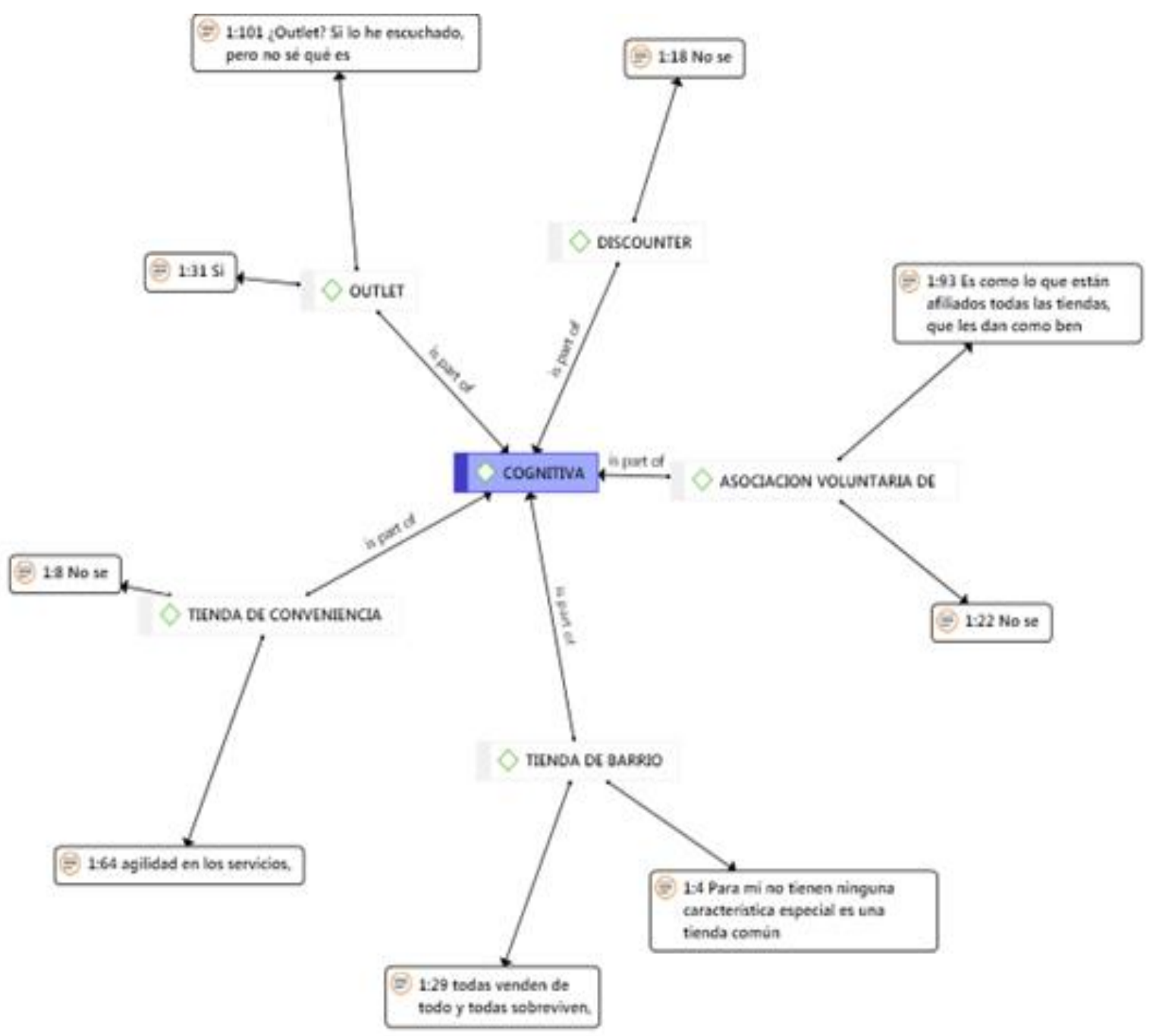

Figura 4: Red semántica cognitiva. Autor: Elaboración propia 
En cuanto al análisis hecho por Atlas Ti para el conocimiento cognitivo se identificaron características que reconocen los consumidores de las diferentes categorizaciones de los establecimientos y sus nombres, generando un resultado negativo, debido a que la mayoría de entrevistados no reconocen los tipos de tiendas o establecimientos; entre los que se encontraban las tiendas de conveniencia, los de descuentos, la asociación voluntaria de tenderos, tienda de barrio y los outlets.

La importancia radica en encontrar los mejores precios y productos de calidad, además de siempre conseguir lo que se está deseando adquirir. Una vez los consumidores responden a estos interrogantes, asignan las categorías diferenciadoras. Gran cantidad de las respuestas respecto a si conocen los outlets dieron como resultado que desconocen de sus características, valores, tal como lo expresó el participante CI 1:101 ¿outlet? si lo he escuchado, pero no sé qué es".

Esto hace referencia en ocasiones al pésimo uso que hacen los interesados en el comercio, confundiendo a los consumidores y creando malestar en su percepción. Cuando se hizo referencia de los de descuentos los participantes no tenían conocimiento de sus características y en su mayoría no han escuchado el término; por lo que conocer esta categoría no influye en la decisión de compra de los consumidores.

Ahora, el análisis mejoró al preguntar por la asociación voluntaria de tenderos; concluyó el participante CI 1:93 "es como lo que están afiliados todas las tiendas, que le dan como beneficios". Otros participantes también aseguraron no conocer el tema como el participante CI 1:22 "no sé". Otra de la categorización que demuestra un conocimiento sobre la clasificación de los establecimientos es la tienda de barrio, la cual es captada comúnmente como lo reflejó el participante CI 1:29 "todas venden de todo y todas sobreviven", estas son entendidas como establecimientos de tradición, las cuales son conocidos por la mayoría de los entrevistados de la zona, además, sus dueños son integrantes de la misma comunidad por lo que es un negocio duradero y de permanencia.

También son percibidas como tiendas que no brindan ningún plus diferenciador a los demás establecimientos, tal y como lo aseguró el participante CI 1:4 "para mí no tienen ninguna característica especial es una tienda común".

Por último, las tiendas de conveniencia las cuales, también, hacen parte del estudio los participantes no tenían el concepto claro, un participante respondió CI 1:8 "no se" y el participante considero que estas se basaban en la prontitud del servicio, CI 1:64 "agilidad en los servicios". Esta última respuesta guiada en lo que la palabra "conveniencia" podría significar en la categorización de los establecimientos, demostró que no existe un conocimiento claro de qué productos se pueden encontrar, qué cambios presentan en sus precios y en cuál es la razón de ser de esta clase de establecimiento.

Para concluir el análisis cognitivo, falta conocimiento en la categorización de los establecimientos en general de la 
población de suba de los estratos 3 y 4 . no consideran las diferencias y desconocen su objetivo comercial, su oferta de valor, precios, servicios y demás. Por lo anterior, no tienen un lugar de preferencia al hacer sus compras más que el encontrar lo que desean a un precio justo y de fácil acceso para el consumidor.

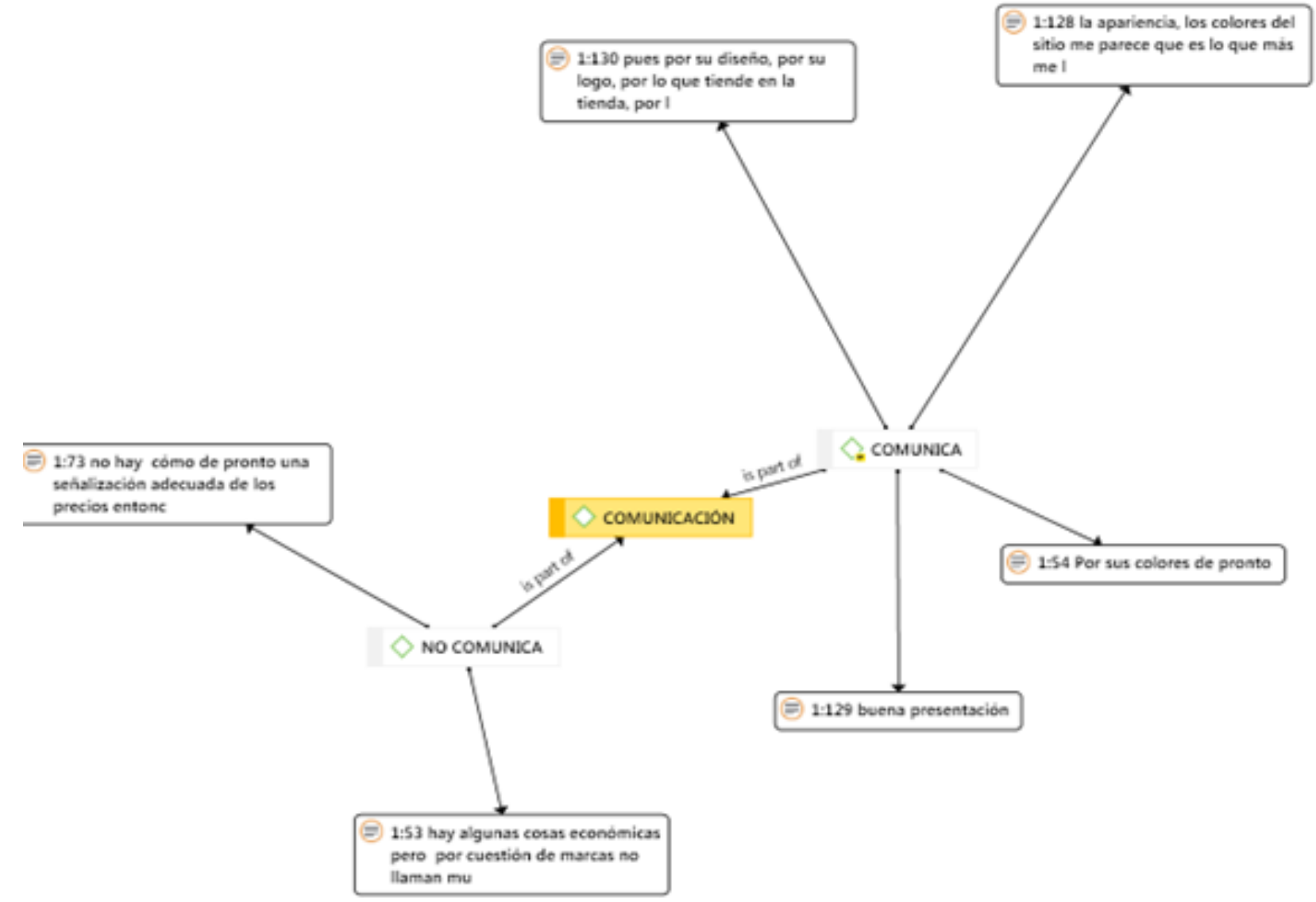

Figura 5: Red semántica comunicación.

Autor: Elaboración propia

Respecto a la codificación de la red semántica de comunicación, se dan a conocer aspectos importantes y relevantes sobre la categorización de los factores claves: comunicación y la no comunicación.

En comunicación se observa aspectos de importancia como: "buena presentación" CL 1:129 indicando el merchandising que se requiere de parte de establecimientos donde se ofrecen servicios, además hay que agregar lo concerniente al factor sensorial, donde los colores, música diseño y demás, son fuente de agrado y atracción de los establecimientos, tal como se lee en esta entrevista: "Por sus colores de pronto" CL 1:54 y "La apariencia de los colores del sitio me parece que es lo que más me llama la atención" CL 1:128 y para contextualizar y dar un análisis general en cuanto a la categorización un aspecto relevante registrado por varios de los entrevistados, tenemos: " pues por el 
diseño, por el logo y por lo que tiene la tienda" CL 1:130.

En cuanto a la categorización de no comunicación, se trae a colación dos aspectos importantes los cuales lo indicaron los entrevistados y dado a las opiniones más importantes de las entrevistas: "Hay algunas cosas económicas, pero por cuestión de marcas no llaman mucho la atención" CL 1:53 "No hay cómo de pronto una señalización adecuada de los precios" CL 1:73.
Sobre estas dos opiniones, se identifica que las marcas no sean tan conocidas es desfavorable para los establecimientos de descuento como D1, Ara y Justo y Bueno, Outlets, tiendas de barrio, entre otros, a pesar de que los precios son bastante favorables para el consumidores, muchos de los participantes dieron a conocer que la marca del producto no tiene notoriedad., considerándose un punto negativo.

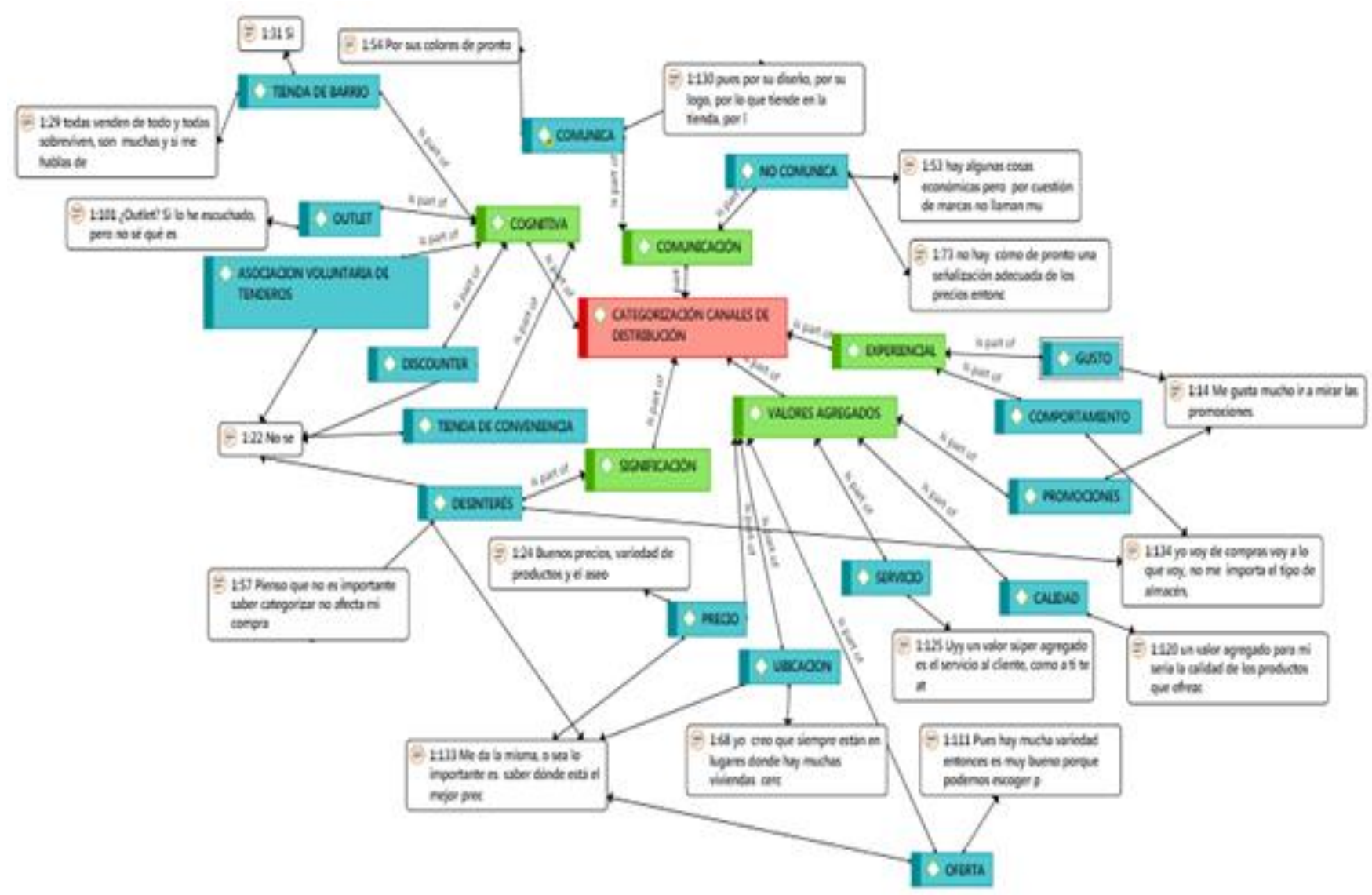

Figura 6: Red semántica general.

Autor: Elaboración propia

Respecto al análisis de comunicación es importante resaltar que la población de suba en donde se llevó a cabo las entrevistas, hay dos aspectos importantes dado a que los consumidores ven una parte importante el hecho de que el establecimiento tenga un aspecto llamativo y atractivo, en cuanto a sus colores, ubicación de productos y presentación. 
Por otro lado, a pesar de que estos establecimientos son de bajos costos para el consumidor, sigue siendo importante el respaldo de una marca, como lo indica las respuestas de los participantes el seleccionar un producto, la marca tiene que ver en la decisión final. No obstante, que puede llegar a ser un poco contradictorio que el consumidor quiera precios bajos, buena fachada y marcas reconocidas, dado que el fin de los de descuento es tener bajos precios, o, marcas propias, o, marcas no muy conocidas

Se evidenció en el estudio que muchos de los consumidores en el momento de adquirir un producto no tienen catalogado los establecimientos para dirigirse a realizar la compra de un producto o servicio, consideran que hay otras características que influyen más a la hora de comprar, como, por ejemplo: el precio, la calidad, la presentación, la ubicación, la oferta, su variedad y el servicio al cliente, ya que son valores agregados para el consumidor. La exhibición de los productos (Merchandising) en este caso es esencial a la hora de comprar, debido a la poca incidencia que presenta la marca tanto del producto como del establecimiento. Hay expectativa sobre el grado de información que existe sobre la clasificación de los establecimientos.

De acuerdo, a los datos arrojados en el proyecto, a través de la codificación abierta, axial y selectiva realizada en Atlas Ti se pudo identificar que las variables dentro del estudio que resaltan las características de la categorización de los canales de distribución son las mismas mencionadas como redes semánticas (cognitiva, comunicación, experiencial, valores agregados y significación).

En cuanto a la categoría de significación fue la clave para evidenciar que por parte de los consumidores no hay interés por la categorización, dejando claro que para ellos como consumidores esos temas son inherentes y no afectan su decisión y experiencia de compra, significando que hay otros valores agregados más importantes para el consumidor, como precio, la calidad, la presentación, la ubicación, la oferta, su variedad y el servicio al cliente.

Así se pudo comprender y conocer los motivos y cualidades que son más importantes para tomar la decisión de dirigirse a uno $\mathrm{u}$ otro canal, $\mathrm{o}$, establecimiento. Debido a que en la actualidad existe una amplia variedad de establecimientos, fue menester conocer que diferencia estratégica permite la decisión de compra por parte de los consumidores.

\section{Conclusiones}

El análisis general de la red semántica cognitiva se basa en la unión que esta tiene con la red semántica de significación, cuyo resultado muestra un desinterés en conocer la categorización por parte de los participantes. Estas se unen por la respuesta "no se" que predominó cuando se interrogaba acerca del conocimiento sobre los de descuentos, 
tiendas de conveniencia y la asociación voluntaria de tenderos. Las respuestas señaladas, demuestran la falta de importancia en cuanto al conocimiento de la categorización de establecimientos por parte de los consumidores; estos basan sus compras en factores personales como el precio, cercanía, amistad, relaciones interpersonales, y encontrar lo que se está buscando.

Ahora bien, una vez se reconoció la conexión entre "no se" y el desinterés, este último continúa su relación con los valores agregados cuya red semántica resalta: ubicación, precio y oferta; estas se complementan con el gusto y comportamiento de los individuos quienes muestran esta conducta en la red experiencial. De esta forma todas tienen reciprocidad y coherencia entre lo que se quiso estudiar del consumidor y la respuesta a la incógnita que esto generó para continuar con el estudio de categorización de establecimientos en Colombia, específicamente en Bogotá en los estratos 3 y 4 del sector de suba.

El análisis de las redes semánticas permitió visualizar que los consumidores no conocen la categorización de los establecimientos y tampoco muestran interés en conocerla, su interés se enfoca en los valores agregados que le ofrecen las diversas superficies. Los valores agregados más significativos fueron: el precio con relación a la calidad y el servicio como características fundamentales tomada como referencia al momento de dirigirse a un canal. Los establecimientos de mayor percepción y aceptación fueron las Tiendas de Barrio y Outlets, sin embargo, denotan desconocimiento profundo en sus objetivos comerciales.

Hay un desconocimiento de las características, valores y servicios que ofrecen establecimientos tan importantes como las tiendas de conveniencia, de descuento, asociación voluntaria de tenderos. Lo anterior, pone de manifiesto la falta de mercadeo en cuanto a una comunicación asertiva hacia el consumidor final.

\section{Agradecimientos}

El trabajo realizado por las personas que se integraron profesionalmente a esta investigación le manifestamos un agradecimiento muy fuerte y profundo por la dedicación desinteresada para el cumplimento de los objetivos propuestos, dichas personas son: Ana, Yessica, Camilo, Daniela C.

\section{Referencias}

America Retail. (01 de 08 de 2019). Colombia: El mundo del Retail está cambiando y cambiará más, ¿estamos listos para ello? Obtenido de https://www.americaretail.com/colombia/colombia-el-mundo-delretail-esta-cambiando-y-cambiara-masestamos-listos-para-ello/

America Retail. (03 de 08 de 2019). Colombia: Estos son los 10 supermercados que más venden en el país. Obtenido de https://www.america-

retail.com/colombia/colombia-estos-son-los10-supermercados-que-mas-venden-en-elpais/

Andjelkovic, A., Barac, N., \& Radosavlje, M. (2017). Analysis of Distribution Channels' Successfulness -The Case of the Retail Chains in the Republic of 
Serbia. Economic Themes, 4(55), 501-519. doi:10.1515/ethemes-2017-0028

Capriotti, P. (1992). La imagen de la empresa: estrategia para una comunicación integrada.

DANE. (2005). BOLETÍN: Censo General 2005 Perfil Localidad Suba - Bogotá. Bogotá: DANE. Obtenido de https://www.dane.gov.co/files/censo2005/per files/bogota/suba.pdf

DRAE. (s.f.). ReaL Academia española. Obtenido de Real Academia española Web site:

http://www.rae.es/recursos/diccionarios/drae

Fenalco. (2012). Fenalco. Obtenido de Fenalco Web site: http://www.fenalco.com.co/contenido/2852/ Los $\% 20$ cambiantes $\% 20 \mathrm{~h} \% \mathrm{C} 3 \%$ A 1 bitos $\% 20$ del $\% 20$ consumidor $\% 20$ colombiano

Frazier, G. (1999). Organizing and Managing Channels of Distribution. Journal of the Academy of Marketing Science (27), 26-240. doi:10.1177/0092070399272007

Frederick Ross, D. (2012). Distribution:Planning and control. Library of congress.

IBM. (2012). Retail 2020: Reinventando la distribucion minorista. New Yorik: IBM Corporation.

Kantabutra, S. (2011). Examining store manager effects in consumer and staff satisfaction: Evidence from Thailand. Journal of Retailing and Consumer Services (18), 46$97 . \quad$ Obtenido de https://www.researchgate.net/publication/22 2560446_Examining_store_manager_effects _in_consumer_and_staff_satisfaction_Evide nce_from_Thailand

Kotler, P., \& Armstrong, G. (2003). Fundamentos de Marketing. Ciudad de mexico: Pearson.
Lazar Knuk, L., \& Schiffman, L. (2005). Comportamiento del consumidor. Pearson Educación.

Molero, V., \& Rivero, J. (2009). Conducta del consumidor: Estrategias y políticas aplicadas al marketing. Madrid: ESIC Editorial.

Real Aacademia Española. (04 de 08 de 2019). Consumidor. Obtenido de https://dej.rae.es/lema/consumidor-ra

Sainz de vicuña Ancin, J. (1996). Comercio tradicional opciones estrategicas. Comercio y consumo, 29.

Sainz de Vicuña Ancín, J. (2000). La distribución comercial: opciones estratégicas. Madrid: ESIC Editorial.

Sampieri, R., Collado, C., Lucio, P., \& Perez, M. (1998). Metodologia de investigacion. Mexico: McGraw Hill.

Secreataría Distrital de Planeación de Bogotá. (02 de agosto de 2019). SUBA 2017. Diagnóstico de los principales aspectos territoriales, de infraestructura, demográficos y socioeconómicos 2017. Obtenido de file:///C:/Users/coord.mercadeo3/Downloads /dice073-monografiasuba-2017_vf.pdf

Secretaría Distrital de Planeación de Bogotá. (02 de agosto de 2019). Análisis demográfico y proyecciones poblacionales de Bogotá. Obtenido de http://www.sdp.gov.co/sites/default/files/de mografia_proyecciones_2017_0_0.pdf 